\title{
In Dedication to Adam P. Showman
}

\author{
Yohai Kaspi ${ }^{1}$
}

Received: 10 March 2021 / Accepted: 11 March 2021 / Published online: 14 April 2021

(c) The Author(s), under exclusive licence to Springer Nature B.V. 2021

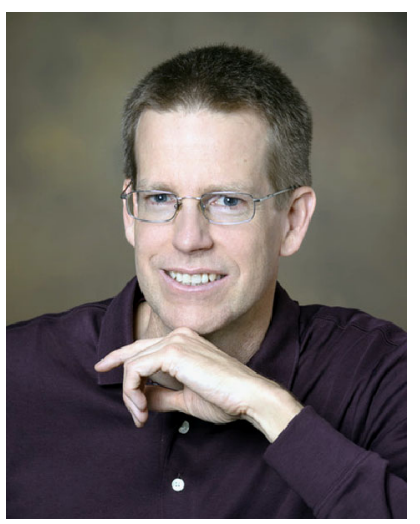

Adam Showman (1968-2020)

This collection is dedicated to the memory of Adam P. Showman, a creative thinker, brilliant scientist, pioneer and leader in the study of the diversity and dynamics of planetary atmospheres.

Son to Dinah and Pete Showman and brother to Ken, Adam grew up in Cupertino, California, and from a young age was passionate about science and art, particularly painting. He liked painting the outdoors, people and stellar objects with a special talent that gave his paintings a 3D perspective. As a child he liked collecting rocks and was enthusiastic about observing the moon through his backyard telescope-an emerging planetary scientist. Drawn to both art and science he entered Stanford University in 1987 eventually deciding to major in Physics, in which he excelled, leaving art as a hobby. Before his senior year he spent a summer at Caltech studying Earth's moment of inertia and its relationship to plate orientation, a project that became his senior thesis and strongly influenced his choice to focus on Planetary Science.

Adam was accepted to the Planetary Science graduate program at Caltech, but deferred graduate school for a year to pursue another passion-Chinese culture, which he fell in love with as a teenager during a family trip to China. Adam spent most of a year teaching English at the University of Geosciences in Wuhan, China, a period which profoundly influenced his perspective throughout his adult life. At Caltech Adam planned to work on data coming from the Mars Observer mission, but when the spacecraft was lost due to a technical failure, he changed course and turned to atmospheric dynamics of giant planets, which was a timely topic prompted by the Galileo spacecraft's arrival at Jupiter in 1995. Adam's PhD work combined interpretation of the Galileo entry probe data, focusing on the dynamics and thermal structure of the atmosphere, and work on the orbital and thermal evolution of Ganymede studying how tidal heating may have restructured the moon's surface. This rare

Understanding the Diversity of Planetary Atmospheres

Edited by François Forget, Oleg Korablev, Julia Venturini, Takeshi Imamura, Helmut Lammer and Michel Blanc

Y. Kaspi

1 Dept. of Earth and Planetary Sciences, Weizmann Institute of Science, Rehovot, Israel 
combination of expertise in both atmospheric dynamics and planetary geophysics was an early indication of Adam's scientific breadth, and were two themes he continued to work on simultaneously throughout his career.

Following his $\mathrm{PhD}$, Adam began a postdoc at the University of Louisville where he was introduced to 3D numerical modeling of giant planets. He employed this tool to explain in detail the flows around Jupiter's hotspots that were recently discovered by the Galileo probe. During this time, the new field of exoplanets was developing rapidly, and Adam became interested in the possible dynamics on these planets. While completing a second postdoc at NASA-Ames, Adam developed, together with Tristan Guillot, the first theory for the atmospheric circulation on a tidally-locked exoplanet. This theory served as a guideline for observers, and indeed several years later their predictions were verified by transit observations of the shift in position of the sub-solar hotspot. This work gave Adam international recognition and pioneered the study of exoplanet atmospheric dynamics.

Adam moved to Tucson in 2001 as an Assistant Professor at the University of Arizona, where he established a new area of research, focusing on planetary atmospheric dynamics, dividing his time between exoplanet atmospheres, giant planet atmospheres and icy satellite research. Adam was a dedicated teacher and mentor, and developed new textbook-level atmospheric dynamics courses for his students. His ability to generalize terrestrial geophysi-

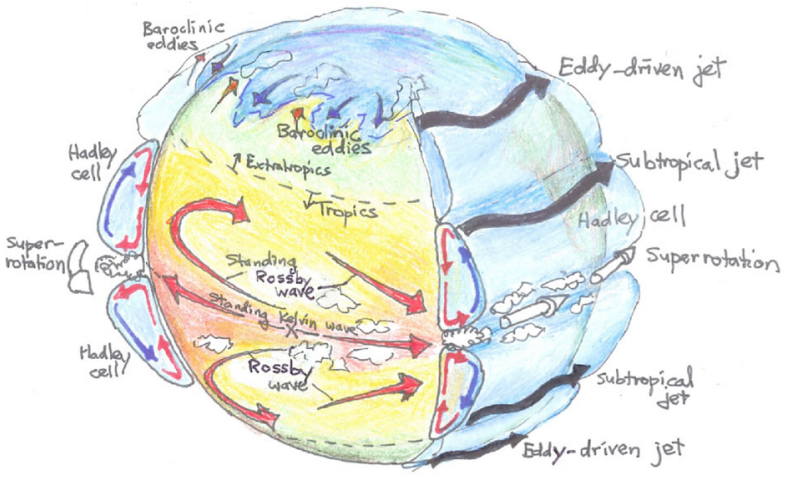

Adam's hand-drawn sketch of the atmospheric general circulation that he made for one of his many review articles. cal flow concepts to other planetary systems was unique and partly what made him the world leader in planetary atmospheric dynamics. His clear and cohesive writing reached well beyond his classes and lectures, producing a dozen review articles and book chapters that will continue to serve as resources for students and researchers alike.

As the field of exoplanets expanded Adam became the leading figure for insight into the atmospheric dynamics of these planets. He had an excellent grasp of theory, numerical modeling and observations and he became the go-to person for both observers and theorists. Extending his initial interest in dynamics, he developed new models, including the detailed radiative properties of these planets, allowing direct connections between his work and the immense growth of observations. He expanded his research to all types of known exoplanet atmospheres including both terrestrial and giant planets, tidally-locked and freely-spinning, over a wide range of atmospheric and orbital configurations, and pioneered the study of dynamics of brown dwarf atmospheres.

In parallel, Adam continued his pursuit of understanding the dynamics on the giant planets of our solar system. He was deeply involved during the early 2000s in the transition of the field from more conceptual studies to using general circulation models of various levels of complexity. This allowed him to establish some fundamental concepts regarding the role of rotation, interior convection, water-vapor and thermal forcing in driving the circulation 
on giant planets. In 2010, he and PhD student Yuan Lian presented the first model that could consistently reproduce the atmospheric circulation on all four giant planets. Alongside his theoretical work, Adam contributed to space mission data interpretation. His group provided the first high-resolution maps of Jupiter's cloud-level winds from the Cassini measurements, and he was involved in developing the methods used to employ the Juno and Cassini gravity measurements to probe the deep atmospheric dynamics of Jupiter and Saturn.

Throughout all the years of his intense scientific activity, Adam found time for his spiritual side through painting, swimming, meditation and studying Chinese culture. Through marriage to Lijie Han and frequent trips to China he became fluent in Chinese. In recent years he spent several hours a day diligently studying the language, including calligraphy, and speaking and writing it to the level of a native, exemplified by his seminars delivered in fluent Chinese while at Peking University. The tall, blond American engaging in intense conversations in Chinese on the streets of Beijing was always accepted with awe and enthusiasm wherever he went. This passion led him to spend most summers of the past decade at the Atmospheric and Oceanic Sciences Department at Peking University, forging many collaborations there. Adam's ability to connect culturally was unique and earned him many friends worldwide. He described his summers in China as extremely influential periods that he cherished.

Adam's quest to understand planetary atmospheres was an expression of his passion to understand nature around him, which he embraced through scientific research, art, and travel. He enjoyed the outdoors and often went on trips across the western US with his daughter Arwen. Explaining, not just observing, was his nature, and this was a powerful driver in his scientific and personal activities. Beyond his scientific achievements, for which he was recognized in 2019 as a fellow of the American Geophysical Union, he demonstrated how great science can be done with kindness, humor and modesty. He paved a path and was a teacher and mentor to many of us following him in a quest to better understand the diversity of planetary atmospheres. His life ended prematurely, but in his time he left a multitude of contributions, a payload of ideas and a generation following his footsteps in a field he pioneered. He will be greatly missed.

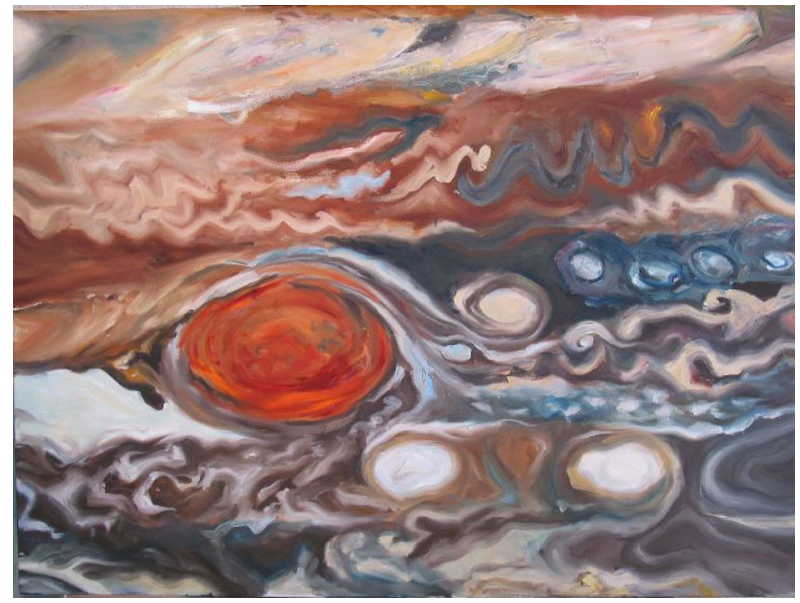

(C) Adam Showman

Publisher's Note Springer Nature remains neutral with regard to jurisdictional claims in published maps and institutional affiliations. 